\title{
PROCESSO DE CONSTRUÇÃO DE INDICADORES EM SAÚDE AUDITIVA
}

\section{Process for constructing indicators in hearing health}

\author{
Luiza Augusta Rosa Rossi-Barbosa (1), Sandra Maria de Freitas Villela Vieira (2), \\ Cláudia Regina Taccolini Manzoni ${ }^{(3)}$, Antônio Prates Caldeira ${ }^{(4)}$, Rodrigo Honorato-Marques ${ }^{(5)}$, \\ Jeniffer Araújo Ribeiro ${ }^{(6)}$, Reila Freitas Silva ${ }^{(7)}$
}

\section{RESUMO}

Objetivo: avaliar a adequação de um instrumento, que está em fase de implantação, elaborado pela Secretaria Municipal de Saúde de São Paulo quanto ao serviço de saúde e uso da prótese auditiva. Métodos: instrumento composto por 17 perguntas que avaliam o serviço e a satisfação do usuário. A avaliação do serviço refere ao atendimento de forma geral, o tempo de espera pelo aparelho, e o(s) retorno(s) após recebimento do aparelho. A satisfação do usuário avalia o tempo de uso do(s) aparelho(s) durante o dia e em quais momentos na vida do paciente o seu uso trouxe melhora. Foi aplicado em 17 sujeitos, com idades entre 21 a 91 anos, média de 61,8 anos. Onze eram do sexo masculino. Resultados: no indicador para avaliação no serviço sugere valorar sobre testagem com mais de um aparelho. No indicador da satisfação há necessidade de modificar a pontuação quanto ao tempo de uso da prótese e quanto à melhora em determinados locais, há ainda necessidade de acréscimo de local de uso do aparelho. Conclusão: faz-se necessária a reformulação e reavaliação do instrumento apresentado. Apesar da limitação devido ao pequeno número de indivíduos pesquisados, verificou-se que os respondentes estão relativamente satisfeitos, porém há queixas quanto à adaptação da prótese auditiva.

DESCRITORES: Indicadores de Serviços; Perda Auditiva; Satisfação do Paciente

(1) Fonoaudióloga; Professora dos Cursos de Odontologia e Medicina da Universidade Estadual de Montes Claros, Unimontes, Montes Claros, MG, Brasil; Mestranda em Ciências da Saúde pela Universidade Estadual de Montes Claros.

(2) Fonoaudióloga da Secretaria Municipal da Saúde de São Paulo, Brasil; Especialista em Linguagem pelo CEFAC Pós-graduação em Saúde e Educação; Especialista em Saúde Coletiva pelo Conselho Federal de Fonoaudiologia; Coordenadora da Área Técnica de Saúde da Pessoa com Deficiência da Coordenação de Atenção Básica, Secretaria Municipal de Saúde de São Paulo.

(3) Fonoaudióloga da Secretaria Municipal da Saúde de São Paulo, Brasil; Especialista em Audiologia e em Saúde Coletiva pelo Conselho Federal de Fonoaudiologia.

(4) Médico; Professor do Curso de Medicina e do Mestrado em Ciências da Saúde da Universidade Estadual de Montes Claros, Unimontes, Montes Claros, MG, Brasil; Doutor em Ciências da Saúde pela Universidade Federal de Minas Gerais.

(5) Aluno do Curso de Graduação em Medicina da Universidade Estadual de Montes Claros, Unimontes, Montes Claros, MG, Brasil.

(6) Aluna do Curso de Graduação em Medicina da Universidade Estadual de Montes Claros, Unimontes, Montes Claros, MG, Brasil.

\section{INTRODUÇÃO}

No Brasil, o Ministério da Saúde instituiu em 2004, por meio da Portaria GM/MS no. 2.073, a Política Nacional de Atenção à Saúde Auditiva. Posteriormente foram criados mecanismos para a estruturação e operacionalização das Redes Estaduais, organizando ações na atenção básica e estruturação de serviços de média e alta complexidade, definindo quantitativos de serviços e suas atribuições. Desde então, Secretarias de Estado têm encaminhado suas redes para habilitação de serviços junto ao Ministério da Saúde.

A constituição destas redes possibilitou um grande avanço no aprimoramento das ações de saúde auditiva do Sistema Único de Saúde (SUS), na medida em que propôs a organização de uma

(7) Aluna do Curso de Graduação em Medicina da Universidade Estadual de Montes Claros, Unimontes, Montes Claros, MG, Brasil.

Conflito de interesses: inexistente 
rede hierarquizada, regionalizada e integrada aos diversos serviços de saúde, contemplando ações de promoção e proteção, diagnóstico, seleção, fornecimento de aparelho auditivo e reabilitação ${ }^{1}$.

Pacientes com problema de audição entram nos serviços de saúde auditiva com o objetivo de melhorar sua função auditiva e consequentemente seu desempenho comunicativo. Quando o tratamento é fornecido, como a concessão do Aparelho de Amplificação Sonora Individual (AASI) ou o serviço de reabilitação, ou ambos, a efetividade do tratamento precisa ser avaliada ${ }^{2}$. A avaliação é parte fundamental no planejamento e na gestão do sistema de saúde e um sistema de avaliação efetivo deve reordenar a execução das ações e serviços, redimensionando-os de forma a contemplar as necessidades de seu público, dando maior racionalidade ao uso dos recursos ${ }^{3}$. Deve-se avaliar o que a população pensa, quer e planeja, não se restringindo às queixas, mas analisando amplamente as condições de vida dos sujeitos na busca da sua qualidade ${ }^{4}$.

O acompanhamento de indicadores pode revelar a eficiência da gestão. Na prática, os indicadores podem ser aplicados para avaliar a estrutura, os processos e os resultados dos serviços de saúde. Eles constituem os sensores de como os serviços estão funcionando, acompanhando e orientando todas as ações e podem detectar as possíveis distorções ou dificuldades que um serviço venha a ter ${ }^{5}$.

Nos Serviços de Saúde Auditiva se faz necessário esta análise, daí a relevância de pesquisas na área e da recente implantação, na cidade de São Paulo, de protocolo, elaborado por Vieira e Manzoni, para avaliação e acompanhamento da satisfação do usuário, como estratégia de responsabilização da unidade para com o indivíduo que mora em seu território e avaliação do resultado da política ${ }^{1}$. O instrumento foi mencionado no II Seminário Científico de Políticas Públicas em Saúde Auditiva, que aconteceu em Bauru - SP, em maio de 2008.

Nesse contexto, o objetivo deste trabalho foi analisar a adequação de um instrumento de avaliação do Serviço de Saúde Auditiva e uso do Aparelho de Amplificação Sonora Individual, que está em fase de implantação, elaborado pela Secretaria Municipal de Saúde de São Paulo.

\section{MÉTODOS}

Trata-se de um estudo piloto de corte transversal, descritivo a partir de questionário composto por 17 perguntas sendo que três delas pontuam de 0 a 10 a avaliação do serviço e duas pontuam de 0 a 10 a satisfação do usuário. A avaliação do serviço refere ao atendimento de forma geral, o tempo de espera pelo aparelho, e o(s) retorno(s) ao serviço após o recebimento do aparelho. A satisfação do usuário avalia o tempo de uso do(s) aparelho(s) durante o dia e em quais momentos na vida do paciente o seu uso trouxe melhora. Foram elaborados pontos de corte em conceitos: ruim, regular, bom, ótimo, sendo ruim a pontuação de 0 a 2, regular de 3 a 5 , bom de 6 a 8 e ótimo 9 e 10.

Dos 1.487 sujeitos cadastrados na Secretaria Municipal da Saúde - Montes Claros - MG, de dezembro de 2005 a 05 de março de 2008, foram selecionados 29 deles pelo método aleatório sistemático com intervalos fixos de 50 em 50, todos moradores no município de Montes Claros - MG, com idade acima de 18 anos, e uso de prótese por mais de seis meses. Destes 29, 12 não foram eletivos, devido falecimento, portador de Alzheimer ou não ter encontrado o usuário na residência após três tentativas.

A pesquisa foi, primeiramente, autorizada pelo gestor da Secretaria de Saúde de Montes Claros - MG e posteriormente analisada e aprovada pelo Comitê de Ética da Universidade Estadual de Montes Claros - Unimontes sob o no 1.084 em 6 de julho de 2008.

Os 17 participantes receberam a visita de um dos pesquisadores em suas residências. Foram esclarecidos e informados a respeito da pesquisa, e assinaram o termo de consentimento livre e esclarecido. Após a coleta de dados, os mesmos foram pontuados pelos indicadores propostos pelo próprio instrumento de avaliação e analisados no SPSS versão 11.0 .

A Figura 1 é o modelo de protocolo elaborado por Vieira e Manzoni, e utilizado pela Secretaria Municipal de Saúde de São Paulo. A Figura 2 refere ao indicador.

\section{RESULTADOS}

Dos 17 entrevistados, onze eram do sexo masculino, com faixas etárias variando entre 21 a 91 anos, média de 61,8 e mediana de 62,0 . Foi observado que $13(76,5 \%)$ não estavam usando a prótese no momento da visita residencial. Quando interrogados se estavam usando o aparelho e por quanto tempo, nove indivíduos (52,9\%) responderam que não estavam usando; quatro $(23,5 \%)$ vêm usando menos da metade do dia; três $(17,7 \%)$ disseram que estão usando o dia todo; e um (5,9\%) relatou usar mais da metade do dia.

Seis $(35,3 \%)$ dos respondentes referiram não ter voltado ao serviço de saúde auditiva após receberem o aparelho. Quando questionados sobre o número de próteses testadas, 14 (82,4\%) 


\section{AVALIAÇÃO - SERVIÇO DE SAÚDE AUDITIVA E USO DO AASI}

Nome: Idade:

Cartão SUS: Prontuário: Informante:

Endereço: Telefone:

Serviço de saúde auditiva que forneceu o aparelho auditivo:

Aparelho que recebeu: OD modelo:

OE modelo: marca: marca:

1. Como procurou o serviço de saúde auditiva?

( ) procura espontânea

( ) recebeu orientação para procurar o serviço. De quem?

( ) foi encaminhado formalmente

( ) outros:

2. Aguardou o atendimento em fila de espera? ( ) sim ( ) não

Por quanto tempo? ( ) 0-3 meses ( ) 3-6 meses ( ) 6-12 meses ( ) 12 a 24 meses ( ) 24 meses ou mais

3. Já usava aparelho auditivo antes? ( ) sim （ ) não

4. Testou mais de um tipo/marca de aparelho em cada orelha? ( ) sim （ ) não

5. Já recebeu o aparelho auditivo? ( ) sim （） não

6. Quanto tempo ficou/está esperando pelo aparelho? Contar a partir da primeira consulta na unidade

( )menos de 1 mês ( )1 a 3 meses ( ) 3 a 6 meses ( ) 6 a 12 meses ( )mais de 12 meses

7. Atualmente:

( ) Sente-se suficientemente orientado para usar o aparelho

( ) Tem dúvidas ou dificuldades para usá-lo

8. Está usando o(s) aparelho(s)?

( ) $\operatorname{sim}$

( ) o dia todo

( ) mais da metade do dia

( ) menos da metade do dia. Explique:

( ) não, porque

9. Está satisfeito com o(s) aparelho(s)? ( ) $) \operatorname{sim}(\quad$ ) não Por que?

10. Retornou ao serviço após o recebimento do aparelho

( ) $\operatorname{sim}$

( ) para aprender a cuidar do aparelho (pilha, molde, limpeza)

( ) para terapia (exercícios para audição ou fala) com o fonoaudiólogo

( ) para nova consulta com médico, audiometria e revisão do aparelho

( ) Não Por que?

11. Tem enfrentado dificuldades em relação à manutenção do aparelho?

( ) sim quais:

( ) não 
12. Como foi o atendimento nos setores?

\begin{tabular}{|l|l|l|l|l|l|}
\hline SETOR & Ótimo & Bom & Regular & Ruim & $\begin{array}{c}\text { Não } \\
\text { passou }\end{array}$ \\
\hline Consulta com otorrinolaringologista & & & & & \\
\hline Exame da audição & & & & & \\
\hline Teste de aparelhos & & & & & \\
\hline Fornecimento e adaptação do aparelho & & & & & \\
\hline Serviço social & & & & & \\
\hline Psicologia & & & & & \\
\hline Terapia fonoaudiológica & & & & & \\
\hline Consulta neurologista & & & & & \\
\hline Consulta pediatra & & & & & \\
\hline
\end{tabular}

13.Como você avalia o atendimento no serviço, de forma geral?

( ) Ótimo
( ) Bom
( ) Regular
( Ruim

14. O uso do aparelho trouxe alguma melhora em sua vida:

( ) $\operatorname{sim}$

( ) para conversar com pessoas da família

( ) para conversar com outras pessoas

( ) nas atividades sociais (igreja, festas,...)

( ) no trabalho ou na escola

( ) para ver televisão ou escutar rádio

( ) não sentiu melhora

15. Gostaria de fazer alguma reclamação?

16. Gostaria de realizar algum elogio?

17. Gostaria de realizar alguma sugestão?

Data:

assinatura e carimbo profissional

nome da unidade de saúde

Figura 1 - Protocolo de São Paulo 


\section{INDICADORES A SEREM UTILIZADOS}

1 - Indicador de Avaliação do serviço: Nota máxima: 10 pontos

Somar os pontos das questões 13, 6 e 10

13. Como você avalia o atendimento no serviço, de forma geral? (máximo 3 pontos)

( 3 ) Ótimo

( 2 ) Bom

( 1 ) Regular

( 0 ) Ruim

6. Quanto tempo ficou/está esperando pelo aparelho? Contar a partir da data da primeira consulta. (máximo 3 pontos)
( 3 ) menos de 1 mês
( 3 ) 1 a 3 meses
( 2 ) 3 a 6 meses
( 1 ) 6a 12 meses
( 0 ) mais de 12 meses

10. Retornou ao serviço após o recebimento do aparelho? (máximo 4 pontos)

( 1 ) sim

( 1 ) para aprender a cuidar do aparelho (pilha, molde, limpeza)

( 1 ) para terapia (exercícios para audição ou fala) com o fonoaudiólogo

( 1 ) para nova consulta com médico, audiometria e revisão do aparelho

( 0 ) Não Por que?

2- Indicador de Avaliação da Satisfação do usuário: (nota máxima- 10 pontos)

Somar os pontos das questões 8 e 13

8- Está usando o(s) aparelho(s)? (máximo 5 pontos)

( $) \operatorname{sim}$

( 5 ) o dia todo

( 3 ) mais da metade do dia

( 1 ) menos da metade do dia.

Explique:

( 0 ) não, porque

14. O uso do aparelho trouxe alguma melhora em sua vida: (máximo 5 pontos)

( ) sim

( 1 ) para conversar com pessoas da família

( 1 ) para conversar com outras pessoas

( 1 ) nas atividades sociais (igreja, festas,...)

( 1 ) no trabalho ou na escola

( 1 ) para ver televisão ou escutar rádio

( 0 ) não sentiu melhora

A relação nominal das pessoas entrevistadas, com as respectivas notas deverão ser encaminhados trimestralmente à Área técnica Saúde da Pessoa com Deficiência, via email (smvieira@prefeitura. sp.gov.br), em planilha conforme modelo abaixo

\begin{tabular}{|l|l|l|}
\hline Nome & Indicador- Serviço & Indicador- Usuário \\
\hline & & \\
\hline & & \\
\hline & & \\
\hline
\end{tabular}

Figura 2 - Indicador de São Paulo 
indivíduos disseram ter testado uma única marca e tipo de aparelho, ou seja, testaram apenas aquele que receberam.

A avaliação da satisfação foi realizada em dois níveis: quanto ao serviço e quanto ao uso do aparelho auditivo. Sobre a satisfação do serviço, 11 usuários $(64,7 \%)$ conceituaram como bom e seis $(35,3 \%)$ como regular. Na avaliação da satisfação do uso do aparelho, um $(5,9 \%)$ classificou como ótimo; três (17,6\%) como bom; 10 (58,9\%) como regular; e três $(17,6 \%)$ como ruim. Dez $(58,9 \%)$ estão satisfeitos por terem recebido o aparelho de amplificação sonora individual, mas entre esses, a metade referiu limitação do uso. Portanto, do total, $12(70,6 \%)$ comentaram algum problema para usar o AASI. Sendo que nove $(52,9 \%)$ referiram incômodo devido ao barulho.

Ao pontuar as respostas dos pacientes verificou-se inadequação da questão $n^{\circ}$ 10, referente ao retorno ao serviço, necessitando incluir uma questão nos indicadores de avaliação do serviço para totalizar 10 pontos. As entrevistas realizadas apontaram para a inclusão da questão no 4: "Testou mais de um tipo/marca de aparelho em cada orelha?" Devendo valorar em 01 ponto o sim e zero ponto o não.

No indicador da satisfação do usuário deparouse com dificuldade em pontuar a questão no 8: "Está usando o(s) aparelho(s)?" para aqueles indivíduos usando apenas um AASI, sendo que estes receberam duas próteses. Por essa razão, acredita-se que a pontuação poderia ser diferente.

Devido ao fato de alguns usuários serem aposentados ou estarem trabalhando $(n=7)$, ao questionar se houve melhora de vida com o uso do aparelho (questão no 14), o item melhora auditiva "no trabalho ou na escola", não foi pontuado, diminuindo o escore da satisfação. Observou ser necessário o acréscimo de mais uma opção como resposta.

Apesar de $82,2 \%$ responderem negativamente à pergunta $n$ - 11: "Tem enfrentado dificuldades em relação à manutenção do aparelho?", no decorrer da entrevista seis deles $(35,3 \%)$ referiram problema em relação às pilhas e dois $(11,8 \%)$ tiveram problema com o molde.

Os usuários comentaram um aumento nos gastos mensais com pilhas, por essa razão economizam usando apenas em horários de maior necessidade, como assistir televisão, dirigir, ou então usam apenas uma prótese. Um dos entrevistados comentou não ter nenhuma reclamação a fazer quanto ao aparelho e ao atendimento, mas não está usando a prótese há seis meses por não ter condições financeiras para comprar as pilhas: "Eu ganho pouco" (E 15). Somente um respondente comentou dificuldade em falar ao telefone. Um outro reclamou que a Secretaria da Saúde não está fornecendo os moldes.

Ao perguntar se tem alguma sugestão a fazer, um usuário respondeu que "a pilha deveria ser doada pelo SUS" (E 15). Outro sugeriu que "o governo... deveria fornecer a pilha. Às vezes não uso porque não tenho dinheiro" (E 11). Um terceiro usuário acha que deveria ter a "visita de um profissional de saúde para acompanhamento e promoção de adaptação" (E 5).

\section{DISCUSSÃO}

O presente trabalho apresenta uma importante contribuição quanto ao processo de avaliação de serviços de saúde auditiva para identificação dos fatores que auxiliam para um atendimento de qualidade, principalmente por considerar que existem poucos trabalhos sobre o tema e a inexistência de uniformidade em relação aos instrumentos.

Geralmente, a avaliação e indicadores desses serviços estão voltados para a infra-estrutura e número de procedimentos realizados, não garantindo a qualidade dos mesmos. Há necessidade de desenvolver indicadores que produzam avaliações coerentes para a gestão da Saúde Auditiva e sinalize ao fonoaudiólogo melhor atendimento à população ${ }^{6}$.

Algumas limitações neste estudo deverão ser consideradas devido ao reduzido número de indivíduos. Deve-se levar em conta, todavia, que o principal aspecto foi a avaliação do instrumento e não a avaliação do serviço propriamente dito.

\section{Sobre o indicador e o questionário}

No que diz respeito às questões e indicadores formulados, a escolha para inclusão da pergunta número 4 justifica-se por constar na Portaria 587 Anexo IV ${ }^{7}$, referente à Seleção e Adaptação de Aparelhos de Amplificação Sonora Individual (AASI), a recomendação de selecionar e testar no mínimo três marcas diferentes de AASI. Outra razão foi a de que $82,4 \%$ relataram sobre testagem com uma só marca de aparelho, bem como o fato de $70,6 \%$ queixarem de algum problema para usá-lo, o que pode ter alguma relação com o tipo e/ou marca que está sendo usado.

No indicador da satisfação do usuário deparouse com indivíduos usando apenas um AASI, sendo que estes receberam duas próteses. Propõe-se, portanto, cinco pontos para aquele que usa o dia todo, quatro pontos para aquele que recebeu dois aparelhos, mas está usando o dia todo somente um AASI; três pontos para uso de mais da metade do dia; dois pontos para aquele que recebeu dois 
aparelhos e está usando apenas um mais da metade do dia; dois pontos para quem usa menos da metade do dia; um ponto para aquele que recebeu dois aparelhos e está usando apenas um durante menos da metade do dia; e zero para aquele que não está usando o aparelho auditivo.

Quanto ao questionário, apesar das respostas negativas em relação à manutenção do aparelho, posteriormente houve relatos de problema com as pilhas e moldes. Sugere-se incluir perguntas direcionadas, tal qual explorado numa recente pesquisa em Recife - PE e que constam no levantamento de dados do prontuário dos usuários de prótese auditiva do Instituto Materno Infantil Fernando Figueira ${ }^{8}$.

Em relação ao item melhora auditiva "no trabalho ou na escola", devido ao perfil dos respondentes, houve uma diminuição no escore em relação à satisfação do usuário. Sugere-se acrescentar "....ou outra ocupação".

Sobre a dificuldade em falar ao telefone, pesquisas demonstram que este é um item importante na explicação de baixos índices de satisfação com o aparelho auditivo ${ }^{9,10}$, por ser uma situação na qual as limitações tecnológicas das próteses ficam evidenciadas ${ }^{9}$.

$\mathrm{Na}$ Figura 3 constam as questões e indicadores propostos neste estudo.

\section{Sobre os respondentes}

Em estudo realizado no estado de Tocantins, no período de 2002 a 2005, foram protetizados 1.364 usuários, sendo que a maior parte das protetizações em adultos concentrou-se entre aqueles acima de
60 anos ${ }^{10}$. Na pesquisa sobre a satisfação de 201 usuários do sistema de saúde do exército, acima de 18 anos, a média de idade foi de 72 anos ${ }^{9}$.

Apesar de diferenças metodológicas entre os diversos trabalhos resultarem em índices de prevalência diferentes, num estudo americano aproximadamente $30 \%$ das pessoas com mais de 65 anos de idade e $50 \%$ das pessoas com mais de 75 anos afirmaram ter alguma perda auditiva ${ }^{11}$.

Nesta pesquisa, apenas $17,6 \%$ vêm usando o aparelho auditivo o dia todo; $29,5 \%$ usam de forma irregular e 52,9\% não estão usando. Esses dados diferem do estudo realizado com usuários de próteses auditivas doadas pelo Núcleo de Atenção Médico Integrada, Fortaleza, no qual foram avaliados 20 pacientes na faixa etária de 65 a 92 anos, e 11 deles $(55,0 \%)$ afirmaram usar o aparelho o dia todo, cinco $(25,0 \%)$ usam de 2 a 4 horas ao dia e quatro $(20,0 \%)$ utilizam a prótese metade do dia ${ }^{12}$. Também, diferem da pesquisa realizada no Ambulatório de Audiologia do serviço de Atendimento Fonoaudiológico da Universidade Federal de Santa Maria, na qual dos 31 indivíduos, 18 (58,1\%) faziam uso integral do aparelho, 13 (41,9\%) utilizavam de forma irregular ${ }^{13}$.

A grande maioria das queixas sobre a limitação do uso refere-se a problemas de adaptação e outras devido a problemas econômicos. Em relação à adaptação, o problema pode estar relacionado à falta de testes com diferentes marcas e tipos de AASI e/ou à falta de retornos ao serviço de saúde auditiva. Nesta pesquisa verificou-se que seis deles referiram não ter voltado ao serviço de saúde auditiva após recebimento do aparelho.

\section{PERGUNTAS PROPOSTAS PARA INCLUIR NO QUESTIONÁRIO}

11. Tem enfrentado dificuldades em relação à manutenção do aparelho:

a) Quanto à reposição da pilha?

( ) Sem dificuldade

( ) Dificuldade:

( ) financeira

( ) distância

( ) outras

b) Quanto à reposição do molde?

( ) Sem dificuldade

( ) Dificuldade:

( ) financeira

( ) distância

( ) outras

c) Alguma outra dificuldade? Qual? 


\section{INDICADORES PROPOSTOS NESTE ESTUDO}

1 - Indicador de Avaliação do Serviço: Nota máxima: 10 pontos

Somar os pontos das questões 13, 6, 4 e 10

13. Como você avalia o atendimento no serviço, de forma geral? (máximo 3 pontos)

( 3 ) Ótimo

( 2 ) Bom

( 1 ) Regular

( 0 ) Ruim

6. Quanto tempo ficou/está esperando pelo aparelho? Contar a partir da data da primeira consulta. (máximo 3 pontos)
( 3 ) menos de 1 mês
( 3 ) 1 a 3 meses
( 2 ) 3 a 6 meses
( 1 ) 6a 12 meses

( 0 ) mais de 12 meses

4. Testou mais de um tipo/marca de aparelho em cada orelha?

( 1 ) $\operatorname{sim}(0$ ) não

10. Retornou ao serviço após o recebimento do aparelho? (máximo 3 pontos)

( ) sim

( 1 ) para aprender a cuidar do aparelho (pilha, molde, limpeza)

( 1 ) para terapia (exercícios para audição ou fala) com o fonoaudiólogo

( 1 ) para nova consulta com médico, audiometria e revisão do aparelho

( 0 ) Não. Por que?

\section{2- Indicador de Avaliação da Satisfação do Usuário: (nota máxima- 10 pontos)}

Somar os pontos das questões 8 e 13

8. Está usando o(s) aparelho(s)? (máximo 5 pontos)

( $\quad) \operatorname{sim}$

( 5 ) o dia todo

( 4 ) o dia todo, mas somente um aparelho, apesar de ter recebido duas próteses.

( 3 ) mais da metade do dia

( 2 ) mais da metade do dia somente um aparelho, apesar de ter recebido dois.

( 2 ) menos da metade do dia.

( 1 ) menos da metade do dia somente um aparelho, apesar de ter recebido dois.

Explique:

( 0 ) não, porque

14. O uso do aparelho trouxe alguma melhora em sua vida: (máximo 5 pontos)

( ) $\operatorname{sim}$

( 1 ) para conversar com pessoas da família

( 1 ) para conversar com outras pessoas

( 1 ) nas atividades sociais (igreja, festas,...)

( 1 ) no trabalho, na escola, ou outra ocupação

( 1 ) para assistir televisão ou escutar rádio

( 0 ) não sentiu melhora

Figura 3 - sugestão de perguntas e indicadores para incluir no questionário 
Pacientes que participaram do Programa de Reabilitação Auditiva, desenvolvido na Policlínica Militar de Porto Alegre - RS, apresentaram níveis de satisfação mais elevados. O programa consiste em esclarecimentos sobre exames audiológicos, noções sobre a perda auditiva e suas implicações; possibilidades e limitações do resultado da protetização; e informações sobre características das próteses auditivas. O paciente que decidir continuar no programa realizará testes com diferentes marcas de próteses, seguidos de experiência domiciliar com o modelo escolhido. Durante esse período participa de sessões de aconselhamento, orientações e treinamento de uso e manuseio da prótese auditiva e de estratégias de comunicação. Caso o paciente adquira a prótese e não necessite de acompanhamento imediato, será reavaliado em três meses. Se for confirmada a adaptação à prótese, é solicitado retorno em um ano. Caso contrário, são levantadas as dificuldades e oferecidas novas estratégias, mantendo-se o acompanhamento até a melhor adaptação. Acompanhar o paciente apenas no momento da aquisição do aparelho não é suficiente ${ }^{9}$. A falta deste acompanhamento traz grandes consequências, pois o uso não efetivo do aparelho auditivo compromete a integração social 8. A literatura aponta uma prevalência baixa de uso de AASI entre idosos, sendo o primeiro ano após a aquisição considerado o período crítico de adaptação, dentro do qual há risco de desistência ${ }^{14}$.

Na presente pesquisa, os usuários comentaram um aumento nos gastos mensais com pilhas usando as próteses em horários de maior necessidade ou então usando apenas uma. Estudos revelam que alguns pacientes não têm condições financeiras em adquirir as pilhas ${ }^{8,10,13}$, sendo referida como uma das maiores dificuldades ${ }^{8}$, havendo relatos de não fazer uso das próteses em tempo integral para não gastá-las ${ }^{13}$. Geralmente, a cartela contém seis pilhas e a duração de cada uma é de no máximo 15 dias $^{8}$. Apesar de tais despesas serem incompatíveis com a renda mensal da maioria dos sujeitos, há um alto índice de satisfação, a possível explicação é a de que os indivíduos se sentem gratos pela concessão do aparelho e julgam que arcar com o gasto de pilhas é uma contrapartida mínima frente ao benefício que receberam do Estado ${ }^{10}$.
Os custos monetários envolvidos com o processo de reabilitação interferem em diferentes aspectos como: aceitação, desempenho, benefício e satisfação com o uso das próteses auditivas ${ }^{13}$.

Um dos respondentes reclamou do fornecimento de moldes. A reposição dessa peça é uma questão polêmica na maioria dos estados ${ }^{8}$.

Embora haja problemas em relação ao aparelho auditivo, supõe-se que os altos níveis de satisfação dos indivíduos traduzem a gratidão pelo recebimento da prótese sem qualquer ônus financeiro, portanto não se considerando dignos de qualquer grau de insatisfação, além do receio de não serem mais beneficiados caso não se mostrem agradecidos ${ }^{8,10}$.

Diante da sugestão de um dos respondentes sobre a visita de profissional da saúde para acompanhamento da adaptação, pode-se dizer que é inadmissível conservar o modelo diagnóstico por parte das políticas públicas sem o respectivo tratamento de reabilitação, pois fere os princípios éticos. Porém, de acordo com a nova política, estão previstas medidas de intervenção por meio de ações de promoção de saúde, proteção, tratamento e reabilitação auditiva.

\section{CONCLUSÃO}

Concluiu-se ser necessária a reformulação e reavaliação do instrumento apresentado, devendo incluir uma pergunta no indicador em relação à testagem do aparelho e modificar a pontuação sobre o tempo de uso do AASI. Sugerimos também pequenas modificações no questionário para melhor entendimento aos entrevistados.

Apesar da limitação do estudo, devido ao pequeno número de indivíduos no piloto ora apresentado, verificou-se que os respondentes estão relativamente satisfeitos quanto ao atendimento no serviço e percebe-se que são gratos por terem recebido a(s) prótese(s), porém a maioria relatou não usar o AASI havendo queixas na adaptação, principalmente devido incômodo quanto ao barulho, o que significa que a avaliação do serviço não está relacionada com a satisfação quanto ao uso do aparelho para melhora da saúde auditiva. 


\begin{abstract}
Purpose: assess the adequacy of a questionnaire, which is in the implementation phase, elaborated by the Municipal Department of Health of São Paulo regarding the hearing health service and the use of hearing aids. Methods: the questionnaire consists of 17 questions that assess the service and user's satisfaction. The assessment of the service comprises the medical care in general, waiting time for the hearing aid, and the return(s) after receiving it. User's satisfaction assesses the period of time when the hearing aid is used during the day and at what times in the patient's life its use has brought improvement. It was applied for 17 subjects, ages 21 to 91 years, mean age: 61.8 years. Eleven were male. Results: the indicator for service assessment suggests pondering over testing with more than one device. The indicator for satisfaction suggests the need to modify the score regarding the period of time the device is used and, regarding the improvement in certain places, it is necessary to add the place where the device is being used. Conclusion: it is necessary to reformulate and reevaluate the submitted questionnaire. Despite its limitation due to the small number o studied $f$ individuals, it was found that the respondents were relatively satisfied, although there are complaints as for the adaptability to the hearing aid.
\end{abstract}

KEYWORDS: Indicators of Health Services; Hearing Loss; Patient Satisfaction

\section{REFERÊNCIAS}

1. Almeida SMVT, Manzoni CRT, Tedesco MRM, Leite SHIC. A implantação da política de saúde auditiva no município de São Paulo. Anais do II Seminário Científico "Políticas Públicas em Saúde Auditiva". São Paulo, maio 2008.

2. Cook JA, Hawkins DB. Outcome measurement for patients receiving hearing aid services. Laryngoscope. 2007; 117(4):610-3.

3. PNASS - Programa Nacional de Avaliação de Serviços de Saúde. Resultado do processo avaliativo de 2004-2006. Brasília, 2007. 85 p.

4. Penteado RZ, Servilha EAM Fonoaudiologia em saúde pública/coletiva: compreendendo prevenção e o paradigma da promoção da saúde. Distúrbios da Comunicação. 2004; 16(1):107-16.

5. Soárez PC, Padovan JL, Ciconelli RM. Indicadores de saúde no Brasil: um processo em construção. Rev. Adm. Saúde. 2005; 7(27):57-64.

6. Bevilacqua MC, Melo TM, Morettin M, Lopes AC. A avaliação de serviços em Audiologia: concepções e perspectivas. Rev. Soc. Bras. Fonoaudiol. 2009; 14(3):421-6.

DOI: 10.1590/S1516-18462010005000061

RECEBIDO EM: 17/11/2009

ACEITO EM: 10/03/2010

Endereço para Correspondência:

Luiza Augusta Rosa Rossi-Barbosa

Rua São Marcos, 115 - Todos os Santos

Montes Claros - MG

CEP: 39400-128

E-mail: luiza.rossi@ unimontes.br
7. Brasil. Ministério da Saúde. Portaria no 587 , de 07 de outubro de 2004. [acesso em set 2007] Disponível em: URL: <http://portal.saude.gov.br/ portal/sas/mac/area.cfm?id_area=824>.

8. Teixeira CF. Estudo avaliativo da política de atenção à saúde auditiva: estudo de caso em Pernambuco - Recife. [tese] Recife (PE): Centro de Pesquisas Ageu Magalhães, Fundação Oswaldo Cruz; 2007. $159 \mathrm{f}$.

9. Veiga LR, Merlo ARC, Mengue SS. Satisfação com a prótese auditiva na vida diária em usuários do sistema de saúde do Exército Rev. Bras. Otorrinolaringol. 2005; 71(1):67-73.

10. Carvalho JSA. Satisfação de idosos com aparelhos auditivos concedidos no estado do Tocantins Arq. Int. Otorrinolaringol. 2007; 11(4):416-26.

11. Neves VT, Feitosa MAG. Envelhecimento do processamento temporal auditivo. Psicol Teor Pesq. 2002; 18(3):275-82.

12. Batista, ACM, Sampaio FM. Nível de satisfação dos idosos usuários de próteses auditivas doadas pela APAC-NAMIUNIFOR. Rev. Bras Promoção Saúde. 2005; 18(1):7-10.

13. Freitas CD, Costa MJ. Processo de adaptação de próteses auditivas em usuários atendidos em uma instituição pública federal - parte I: resultados e implicações com o uso da amplificação Rev. Bras. Otorrinolaringol. 2007; 73(6):744-51.

14. Ruschel CV, Carvalho CR, Guarinello AC. A eficiência de um programa de reabilitação audiológica em idosos com presbiacusia e seus familiares. Rev. Soc. Bras. Fonoaudiol. 2007; 12(2):95-8. 